\title{
Parameter Extraction Procedure for Ion Implantation Profiles to Establish Robust Database based on Tail Function
}

\author{
Kunihiro SUZUKI* and Shuichi KOJIMA**
}

\begin{abstract}
We proposed a tail function parameter extraction procedure for the establishment of a robust ion implantation database. We showed that, for the expression of ion implantation profiles, there are many local minimum values set for the third and fourth moment parameters of $\gamma$ and $\beta$ for the Pearson function that comprises the standard dual Pearson and tail functions. We proposed the use of a joined tail function as a mediate function to extract $\gamma$ and $\beta$, and demonstrated that this enables us to extract the parameters uniquely. Other parameters associated with channeling phenomena can also be simply and uniquely extracted by our procedure.
\end{abstract}

Index Terms - Ion implantation, Pearson function, tail function, database

\section{INTRODUCTION}

The ion implantation profile database is important in process simulation for predicting profiles for a vast number of ion implantation conditions used in VLSI processes. The database consists of a parameter value table for a function to fit the experimental data. The accuracy of the simulation depends on the function used. Functions to express ion implantation profiles have also been developed by using Gauss, joined half Gauss [1], Pearson $[2,3]$, dual Pearson $[4,5]$, and tail functions[6-

Manuscript received May 25, 2010; revised Oct. 30, 2010

* Fujitsu Laboratories Ltd., 10-1 Morinosato-Wakamiya, Atsugi 243-01, Japan

** Fujitsu LSI Technology, Fuchigami 50, Akiruno City, Tokyo 197-0833, Japan

E-mail : suzuki.kunihiro@jp.fujitsu.com
9]. It has been demonstrated that the dual Pearson accurately covers the whole ion implantation profile, and it is the standard function implemented in commercial simulators [10]. We pointed out a problem with the dual Pearson regarding uniqueness of the parameter values and demonstrated that the tail function solves the problem [6-8]. The tail function and the dual Pearson function include a Pearson function. Here, we pointed out that it is difficult to obtain a unique parameter set for the third $(\gamma)$ and forth moment $(\beta)$ parameters of this Pearson function. We propose a method of extracting the values of $\gamma$ and $\beta$ ensuring the uniqueness of their values. Finally, we propose an extraction procedure for tail function parameters associated with channeling phenomena.

\section{TAIL FUNCTION}

Here, we briefly explain the tail function [8]. An instance of ion implantation is expressed in the tail function by two components, one for the amorphous part $n_{a}$, and one for the channeling part $n_{c}$, as given by

$$
N(x)=\left(\Phi-\Phi_{\text {chan }}\right) n_{a}(x)+\Phi_{\text {chan }} n_{c}(x)
$$

$n_{a}$ is expressed by a Pearson function $h_{m a}$ with moment parameters $R_{p}, \Delta R_{p}, \gamma$, and $\beta$, given by

$$
n_{a}(x)=h_{m a}(x)
$$

$n_{c}(x)$ is also a Pearson function up to depth $x_{T}$, and is 
the sum of the Pearson and single tail function $h_{T c}$ (we call it the single tail function to distinguish it from the tail function of Eq. (1) in the deeper region, given by

$$
n_{c}(x)= \begin{cases}h_{m c}(x) & \text { for } x \leq x_{T} \\ \kappa\left[h_{m c}(x)+h_{T c}(x)\right] & \text { for } x>x_{T}\end{cases}
$$

Pearson function $h_{m c}$ has the same moment parameters as that of $h_{m a}$. The single tail function is given by

$$
h_{T c}(x)=h_{m c}\left(x_{p}\right) \exp \left[-(\ln \eta)\left(\frac{x-x_{p}}{L}\right)^{\alpha}\right]
$$

where

$$
x_{T}=R_{p}+\Delta R_{p}
$$

and $x_{p}$ is the peak position. $\kappa$ is determined so as to ensure continuity at $x=x_{T}$, and is obtained from the equation

$$
h_{m c}\left(x_{T}\right)=\kappa\left[h_{m c}\left(x_{T}\right)+h_{T c}\left(x_{T}\right)\right]
$$

$\eta$ is arbitrary, but is constant at 1000 here. This function expresses that the concentration at distance $L$ from $x_{p}$ is $1 / 1000$ of the peak concentration of $h_{m c}\left(x_{p}\right) \cdot \alpha$ expresses the shape of the tail function and is exponential like with 1, and Gaussian like with 2 . The parameters $L$ and $\alpha$ are directly related to the shape of the profiles, and a unique parameter set can be expected and demonstrated [6-8].

Our tail function also contains a Pearson function as can be seen in Eqs. (1-3). We showed that parameters $\gamma$ and $\beta$ of the function are difficult to extract uniquely. This is therefore a problem for both the dual Pearson and tail function.

\section{EXTRACTION OF $\gamma$ AND $\beta$ OF PEARSON FUNCTION}

In this section, we discuss the extraction of $\gamma$ and $\beta$ for the Pearson function. We treat profiles in an amorphous layer in order to focus on a Pearson function for the amorphous part of Eq. (2), without considering the channeling profile.

Fig. 1 compares Secondary Ion Mass Spectrometry (SIMS) data and analytical data using a Pearson function. The Pearson function readily reproduces SIMS data that cannot be expressed by the Gauss or joined half Gauss functions, as shown later.

Fig. 2 shows extracted parameters. $R_{p}$ and $\Delta R_{p}$ are almost linearly dependent on energy. $\gamma$ set constant at 1.5, which will be explained later. This means that the profiles are asymmetrical and the concentration near the surface region is high compared to the deeper region. $\beta$ is around 9 and decreases with increasing energy.

The analytical model readily reproduces the SIMS data. However, let us examine the profile and extracted parameters in more detail. The B profiles look comparatively symmetrical at low energies and become asymmetrical with increasing energy. Therefore, $\gamma$ should be near 0 and become smaller with increasing energy. However, the $\gamma$ value extracted is constant at 1.5 , which does not express the qualitative trend of the profiles above. This is a consequence of our extraction method, as shown below.

Fig. 3 shows that various combinations of $(\gamma, \beta)$ can reproduce the SIMS profile, which means that the parameter set of $(\gamma, \beta)$ lacks uniqueness. This represents a fatal problem if we want to establish a robust database. Of course, it is possible to extract decreasing $\gamma$ with increasing energy, as would be

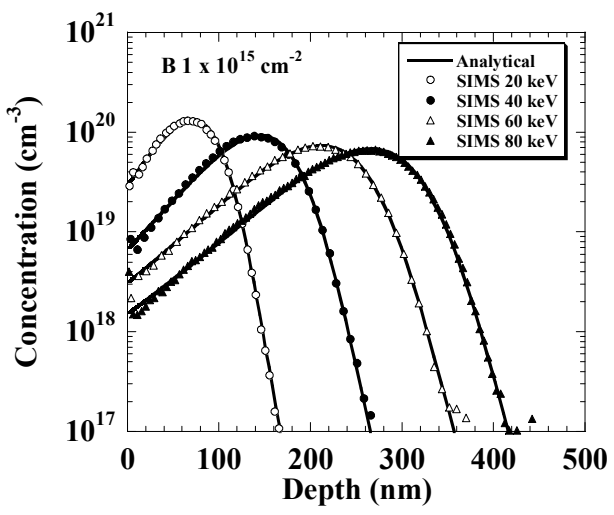

Fig. 1. Concentration profiles of $\mathrm{B}$ ions implanted in amorphous Si. 


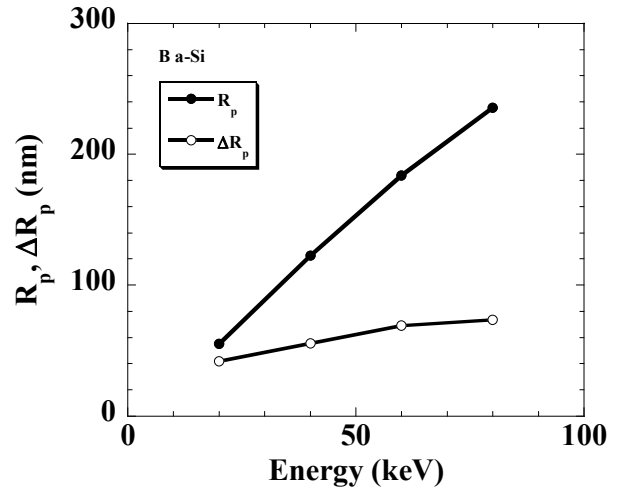

(a)

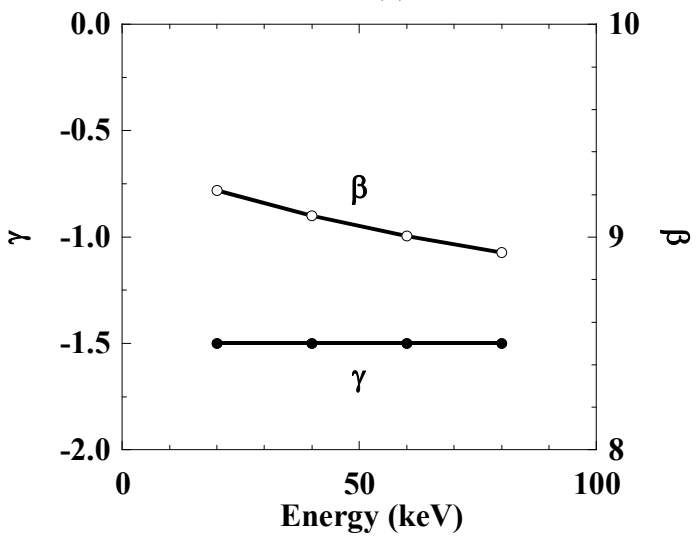

(b)

Fig. 2. Extracted moment parameters for B profiles. (a) $R_{p}, \Delta R_{p}$, (b) $\gamma, \beta$.

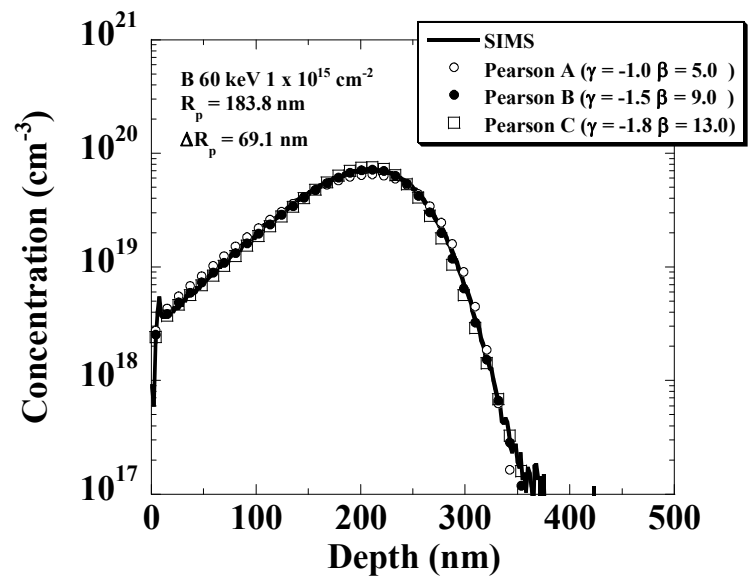

Fig. 3. Reproduction of the SIMS B profile using Pearson functions with various $(\gamma, \beta)$.

expected. However, it is not clear what value of $\gamma$ we should choose. This is the reason we use constant $\gamma$, as shown in Fig. 2(b). In this way, we force the $\gamma$ values to be constant as far as possible, and reproduce the profile by correspondingly tuning the $\beta$ values.

Fig. 4 shows plots of $\left(\gamma^{2}, \beta\right)$ in the $\left(\gamma^{2}, \beta\right)$ plane

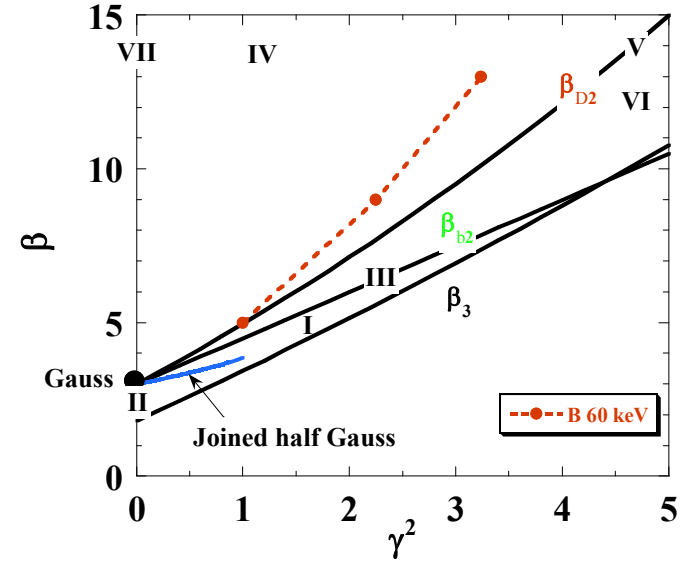

Fig. 4. Plot of extracted $\left(\gamma^{2}, \beta\right)$ in the $\left(\gamma^{2}, \beta\right)$ plane.

for the data in Fig. 3. The regions associated with various Pearson functions [3] are also shown, where

$$
\begin{gathered}
\beta_{D 2}=\frac{3\left(13 \gamma^{2}+16\right)+6\left(\gamma^{2}+4\right)^{\frac{3}{2}}}{32-\gamma^{2}} \\
\beta_{b 2}=\frac{3}{2} \gamma^{2}+3 \\
\beta_{3}=\frac{9\left[6 \gamma^{2}+5+\sqrt{\frac{9}{16} \gamma^{6}+8 \gamma^{4}+25\left(\gamma^{2}+1\right)}\right]}{50-\gamma^{2}}
\end{gathered}
$$

It is expected that the $(\gamma, \beta)$ sets on the line are able to reproduce the SIMS profiles of B ion implanted at 60 $\mathrm{keV}$. This means that the parameters $\gamma$ and $\beta$ interact strongly with each other. Therefore, it is difficult to obtain a unique $(\gamma, \beta)$.

\section{NEW PARAMETER EXTRACTION Procedure using Mediate Function}

In this section, we show that simple Gauss, and joined half Gauss functions have limited values of $\gamma$ and $\beta$. They may be used as mediate functions to extract $\gamma$ and $\beta$. However, they cannot cover whole implantation profiles. Therefore, we extend joined half Gauss functions to joined half tail functions to form a meditate function for the parameters of $n_{a}$, as will be shown later.

First, we briefly study the $\gamma$ and $\beta$ of Gauss and joined half Gauss functions.

It is clear that $(\gamma, \beta)=(0,3)$ for a Gaussian profile. 
Therefore, there is no uniqueness problem with this function.

Next, we investigate $(\gamma, \beta)$ of the joined half Gauss function, given by

$$
N(x)=\left\{\begin{array}{l}
N_{m} \exp \left[-\frac{1}{2}\left(\frac{x-R_{p m}}{\Delta R_{p 1}}\right)^{2}\right] \quad x \leq R_{p m} \\
N_{m} \exp \left[-\frac{1}{2}\left(\frac{x-R_{p m}}{\Delta R_{p 2}}\right)^{2}\right] \quad x>R_{p m}
\end{array}\right.
$$

We define the ratio

$$
\frac{\Delta R_{p 2}}{\Delta R_{p 1}} \equiv r
$$

$\gamma$ and $\beta$ are evaluated by the moments of Eq. 10 and uniquely determined by $r$ as

$$
\begin{gathered}
\gamma=\frac{\sqrt{\frac{2}{\pi}}(r-1)\left[\left(\frac{4}{\pi}-1\right)(r-1)^{2}+r\right]}{\left[\left(1-\frac{2}{\pi}\right)(r-1)^{2}+r\right]^{\frac{3}{2}}} \\
\beta=\frac{\left[3-\frac{4}{\pi}-3\left(\frac{2}{\pi}\right)^{2}\right](r-1)^{4}+\left(9-\frac{20}{\pi}\right)(r-1)^{2}+3 r^{2}}{\left[\left(1-\frac{2}{\pi}\right)(r-1)^{2}+r\right]^{2}}
\end{gathered}
$$

Fig. 5 shows the dependence of $(\gamma, \beta)$ on $r$ for the joined half Gauss function. It is noteworthy that $\gamma$ and $\beta$ change only slightly with $\mathrm{r}$, and only limited values are available for $\gamma$ and $\beta . \Delta R_{p 1}$ and $\Delta R_{p 2}$ are directly related to the shape of the profiles, are uniquely determined, and are almost independent of each other. If $\Delta R_{p 1}$ and $\Delta R_{p 2}$ change continuously with energy, we can expect continuous corresponding changes in $(\gamma, \beta)$, that is, we can expect unique $(\gamma, \beta)$. The limited values of $\gamma^{2}$ and $\beta$ can be evaluated from Eqs. $(12,13)$ as

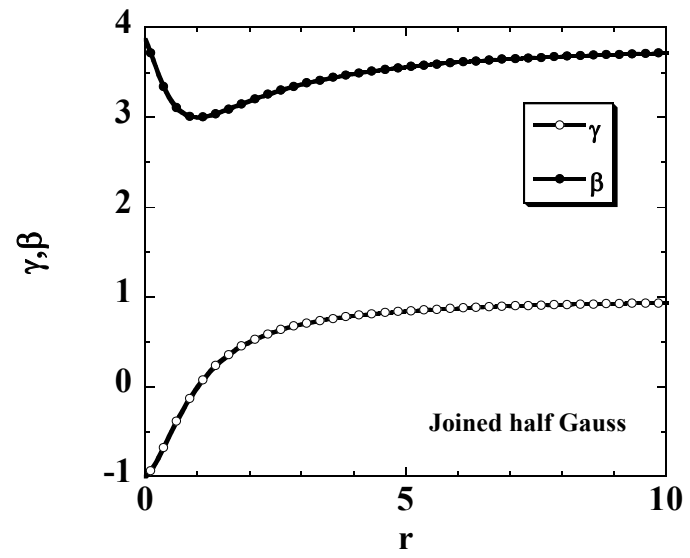

Fig. 5. Dependence of $\gamma$ and $\beta$ on $r$.

$$
\begin{gathered}
\gamma_{\text {min }}^{2}=\gamma(1)^{2}=0 \\
\gamma_{\text {max }}^{2}=\lim _{r \rightarrow 0, \infty} \gamma(r)^{2}=-\left(\frac{\left.\sqrt{\frac{2}{\pi}}\left(\frac{4}{\pi}-1\right)\right)^{2}}{\left.\left(1-\frac{2}{\pi}\right)^{\frac{3}{2}}\right)^{2}}=0.99\right. \\
\beta_{\text {min }}=\beta(1)=3 \\
\beta_{\text {max }}=\lim _{r \rightarrow 0, \infty} \beta(r)=\frac{\left[3-\frac{4}{\pi}-3\left(\frac{2}{\pi}\right)^{2}\right]}{\left(1-\frac{2}{\pi}\right)^{2}}=3.87
\end{gathered}
$$

The joined half Gauss can cover various profiles in amorphous layers $[1,6]$, but cannot cover whole ion implantation profiles, such as that of $\mathrm{B}$ at high energies. Fig. 6 shows SIBM B concentration profile ion implanted at $80 \mathrm{keV}$ and a dose of $1 \times 10^{15} \mathrm{~cm}^{-2}$. The profile is asymmetrical and hence cannot be expressed with Gauss. The joined half Gauss can express asymmetrical profiles, and readily reproduce the SIMS profiles in the deeper region and near the peak concentration region. However, the function cannot express exponential like shape in the surface region. On the other hand, Pearson function readily expresses the profile over whole region. As a consequence, Gauss and joined half Gauss have unique $(\gamma, \beta)$ but cannot express whole ion implantation profiles accurately, while Pearson can express whole ion implantation profiles accurately but have various $(\gamma, \beta)$. 


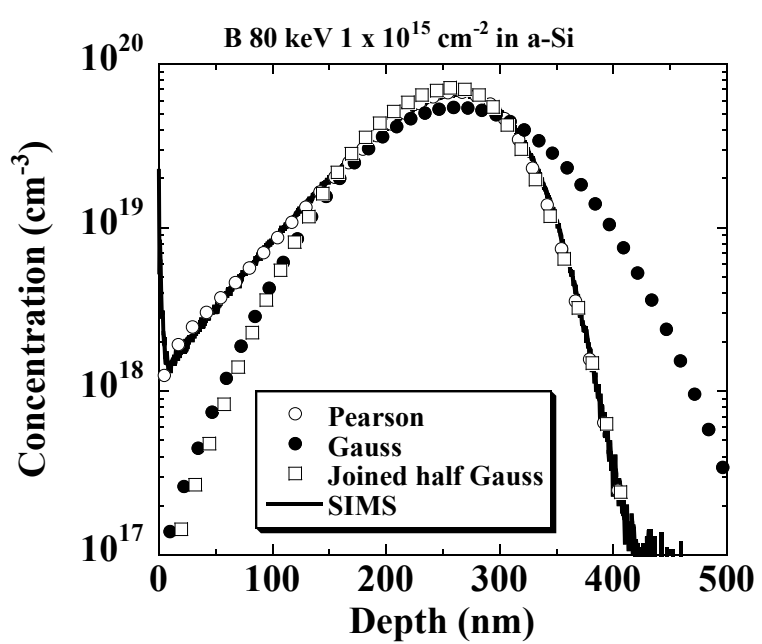

Fig. 6. Comparison of SIMS B profile with fitted analytical Gauss, joined half Gauss, and Pearson functions.

Here, we propose a mediate function to extract moment parameters instead of directly extracting $\gamma$ and $\beta$ from the profile. We propose a joined tail function given by

$$
N(x)=\left\{\begin{array}{l}
N_{m} \exp \left[-\frac{1}{2}\left(\frac{R_{p m}-x}{\Delta R_{p 1}}\right)^{\alpha_{1}}\right] \quad x \leq R_{p m} \\
N_{m} \exp \left[-\frac{1}{2}\left(\frac{x-R_{p m}}{\Delta R_{p 2}}\right)^{\alpha_{2}}\right] \quad x>R_{p m}
\end{array}\right.
$$

$\gamma$ and $\beta$ for this function are evaluated by the moments of Eq. (18) and evaluated also uniquely dependent on $r$, given by

$$
\begin{gathered}
\gamma(r)=\frac{f_{3}(r)-3 f_{1}(r) f_{2}(r)+2 f_{1}(r)^{3}}{\left[f_{2}(r)-f_{1}(r)^{2}\right]^{\frac{3}{2}}} \\
\beta(r)=\frac{f_{4}(r)-4 f_{1}(r) f_{3}(r)+6 f_{1}(r)^{2} f_{2}(r)-3 f_{1}(r)^{4}}{\left[f_{2}(r)-f_{1}(r)^{2}\right]^{2}}
\end{gathered}
$$

where

$$
f_{1}(r) \equiv \frac{-\frac{\Gamma\left(\frac{2}{\alpha_{1}}\right)}{\alpha_{1}}+\frac{\Gamma\left(\frac{2}{\alpha_{2}}\right)}{\alpha_{2}} r^{2}}{\Gamma\left(1+\frac{1}{\alpha_{1}}\right)+\Gamma\left(1+\frac{1}{\alpha_{2}}\right) r}
$$

$$
\begin{array}{r}
f_{2}(r) \equiv \frac{\frac{\Gamma\left(\frac{3}{\alpha_{1}}\right)}{\alpha_{1}}+\frac{\Gamma\left(\frac{3}{\alpha_{2}}\right)}{\alpha_{2}} r^{3}}{\Gamma\left(1+\frac{1}{\alpha_{1}}\right)+\Gamma\left(1+\frac{1}{\alpha_{2}}\right) r} \\
f_{3}(r) \equiv \frac{-\frac{\Gamma\left(\frac{4}{\alpha_{1}}\right)}{\alpha_{1}}+\frac{\Gamma\left(\frac{4}{\alpha_{2}}\right)}{\alpha_{2}} r^{4}}{\Gamma\left(1+\frac{1}{\alpha_{1}}\right)+\Gamma\left(1+\frac{1}{\alpha_{2}}\right) r} \\
f_{4}(r) \equiv \frac{\Gamma\left(\frac{5}{\alpha_{1}}\right)}{\Gamma\left(1+\frac{1}{\alpha_{1}}\right)+\Gamma\left(\frac{5}{\alpha_{2}}\right)} r^{5} \\
\alpha_{2}
\end{array}
$$

Fig. 7 shows the dependence of $\beta$ on $\gamma^{2}$ with $\alpha_{1}$ equal to $1.5,2$ and 2.5, where we assume $\alpha_{2}=\alpha_{1}$ for simplicity. The allowable region is limited with increasing $\alpha_{1}$. The limiting values are also evaluated as

$$
\begin{gathered}
\gamma_{\text {min }}^{2}=\gamma(1)^{2}=\left[\frac{f_{3}(1)-3 f_{1}(1) f_{2}(1)+2 f_{1}(1)^{3}}{\left[f_{2}(1)-f_{1}(1)^{2}\right]^{\frac{3}{2}}}\right]^{2}=0 \\
\beta_{\text {min }}=\beta(1) \\
\gamma_{\text {max }}^{2}=[\gamma(0)]^{2} \\
\beta_{\text {max }}=\beta(0)
\end{gathered}
$$

as shown in Fig. 8.

$\Delta R_{p 1}$ and $\Delta R_{p 2}$ in Eq. (18) correspond to the distance where the concentration is $1 / e^{2}$ times peak concentration. Therefore, it is related to the distance very near the peak region, and hence it is awkward to fit the data. We therefore use the modified relations

$$
N(x)=\left\{\begin{array}{l}
N_{m} \exp \left[-\ln \left(\zeta_{1}\right)\left(\frac{R_{p m}-x}{L_{1}}\right)^{\alpha_{1}}\right] \quad x \leq R_{p m} \\
N_{m} \exp \left[-\ln \left(\zeta_{2}\right)\left(\frac{x-R_{p m}}{L_{2}}\right)^{\alpha_{2}}\right] \quad x>R_{p m}
\end{array}\right.
$$




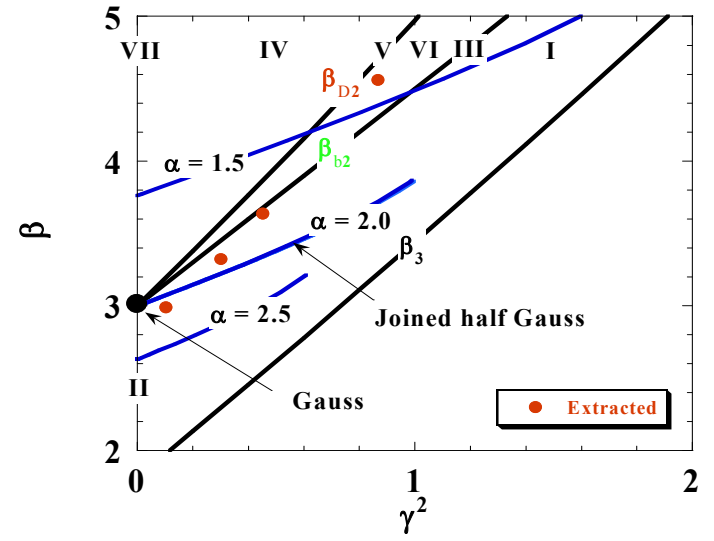

Fig. 7. Dependence of $\beta$ on $\gamma^{2}$ with $\alpha_{1}=1.5,2,2.5$, where we assume $\alpha_{2}=\alpha_{1}$.

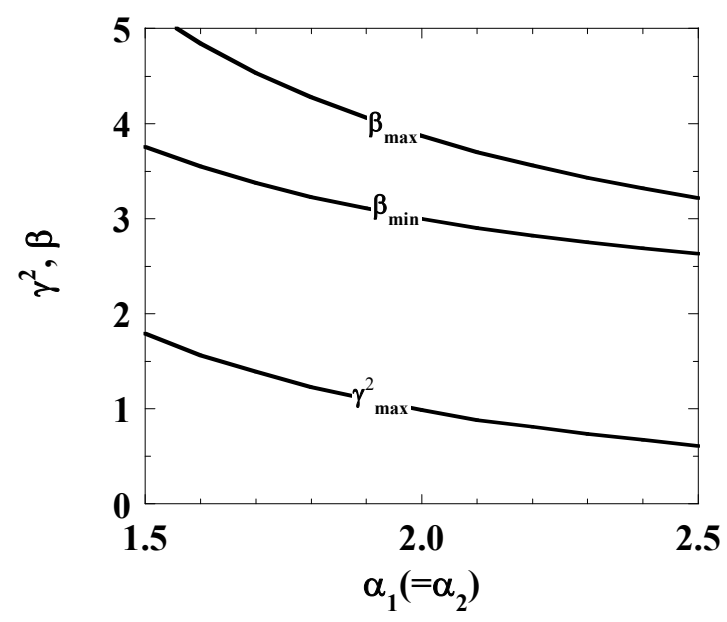

Fig. 8. Dependence of minimum and maximum $\gamma^{2}$ and $\beta$ on $\alpha_{1}$.

$L_{1,2}$ is the distance from the peak position where the concentration is $N_{m} / \varsigma_{1,2}$. Therefore, we can easily fit the theory to the data by inspecting the profiles. $L_{1,2}$ can be related to $\Delta R_{p 1,2}$ as

$$
\Delta R_{p 1,2}=\frac{1}{\left[2 \ln \left(\zeta_{1,2}\right)\right]^{\frac{1}{\alpha_{1,2}}}} L_{1,2}
$$

Fig. 9 shows the joined tail function fitted with SIMS B profiles. We also extract the moment parameters from the joined tail function and generate a Pearson function using the same moments. It should be noted that the joined tail function and Pearson function slightly deviate from each other at high energy. Therefore, we should, at the same time, focus on the Pearson function.

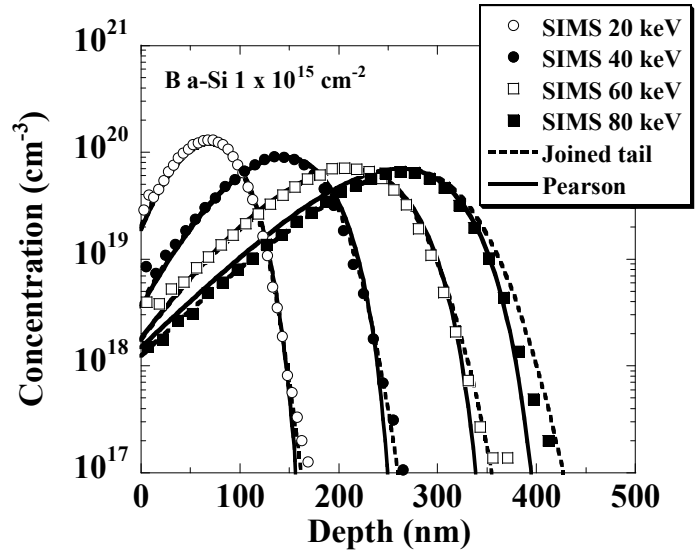

Fig. 9. Comparison of SIMS data with joined tail function, and Pearson function generated by the moment parameters of the joined tail function.

Fig. 10 shows the dependence of the parameters of the joined tail function on energy. $R_{p m}$ and $L_{1}$ increases energy as the energy increases. $L_{2}$ also increases linearly but the dependence is weak compared with $R_{p m}$ and $L_{1} . \alpha_{1}$ decreases with increasing energy, which means that the shape moves from Gaussian like to exponential like. $\alpha_{2}$ is almost constant. These parameters are directly related to the shape of the profiles, and only limited values are available.

Fig. 11 shows the dependence of the moment parameters of the Pearson function on the energy extracted from the joined tail function, acting as a mediate function. $R_{p}$ and $\Delta R_{p}$ increase linearly with increasing energy and $\Delta R_{p}$ is around $1 / 3$ of $R_{p}$. The weak dependence of $\Delta R_{p}$ on energy can be explained by the weak dependence of $L_{2}$ on energy. The resultant $\gamma$ decreases with increasing energy, which is what we would expect. Conversely, $\beta$ increases with increasing energy, which is the opposite trend to that in Fig. 2.

Fig. 7 shows the extracted $\gamma, \beta$ in the $\left(\gamma^{2}, \beta\right)$ plane. $\left(\gamma^{2}, \beta\right)$ exists in the region for Pearson I, III and VI. This also shows the profile at high energy with a highly asymmetric profile that cannot be expressed by the joined half Gauss. 


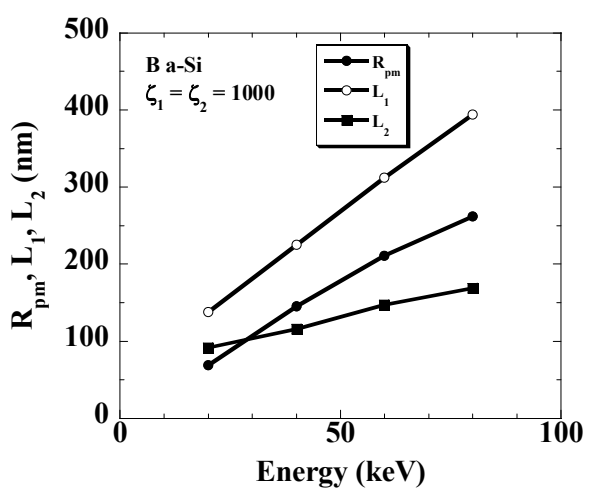

(a)

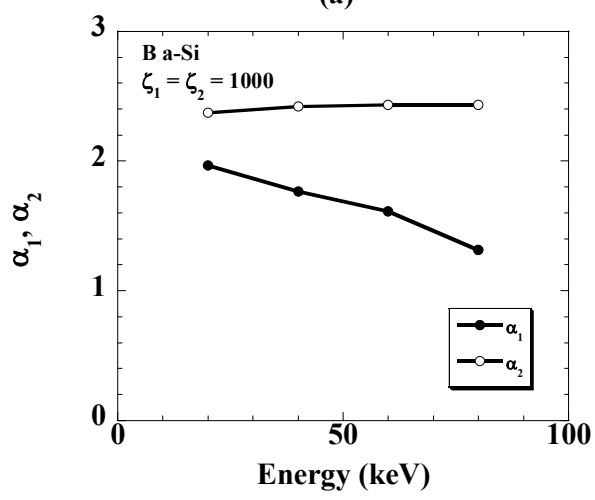

(b)

Fig. 10. Extracted parameters of joined tail function.

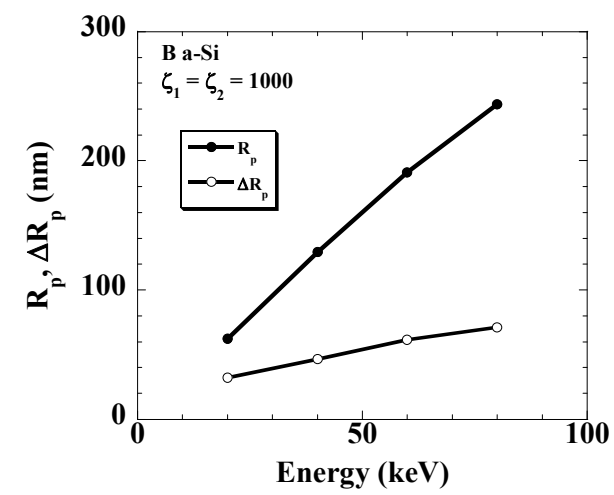

(a)

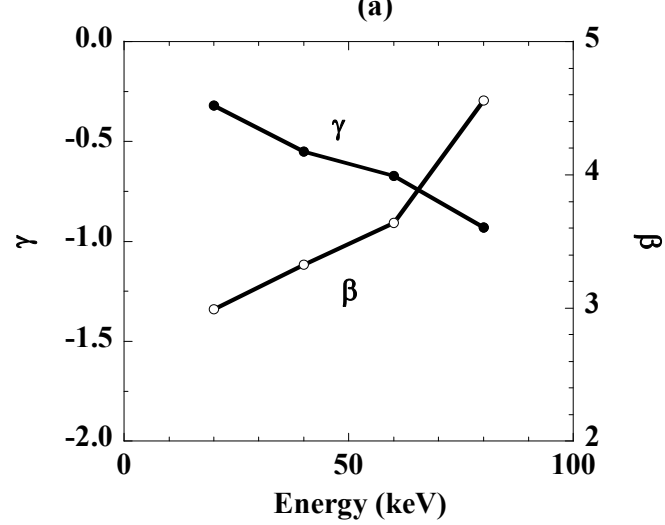

(b)

Fig. 11. Extracted moment parameters evaluated using joined tail function as mediator.

\section{PARAMeter EXtraction OF L AND $\alpha$}

In the previous section, we showed that the parameters of the main part of the tail function, that is, the Pearson function, can be robustly extracted. Now, let us move on to the channeling part of the tail function. The tail function itself is expressed by $h_{T c \_} n_{m 0}(x)$.

$$
h_{T C_{-} n_{m 0}}(x)=n_{m 0} \exp \left[-(\ln \eta)\left(\frac{x-x_{p}}{L}\right)^{\alpha}\right]
$$

We simply use this function and extract $n_{m 0}, L$ and $\alpha$.

We can use $L, \alpha$ as the final parameters, but $n_{m 0}$ is a tentative parameter, which should be converted to $n_{m}$. We can evaluate $\kappa$ using Eq. (6), which is determined from $L, \alpha$ independent of $n_{m}$. We express the implemented tail function as $h_{T C}(x)$ and express its peak concentration as $n_{m}$. In the deep $\mathrm{x}$ region, the following should hold

$$
\kappa h_{T C_{-} n_{m}}(x)=h_{T C_{-} n_{m 0}}(x)
$$

We can obtain $n_{m}$ as

$$
n_{m}=\frac{n_{m 0}}{\kappa}
$$

Integrating this over depth, we can obtain the channel dose

$$
\Phi_{\text {chan }}=\int n_{c}(x) d x
$$

Fig. 12 compares the SIMS As profile with the fitted tail function, where we extract the parameters as follows.

The reliable dynamic range of the SIMS profile in the region from the peak towards the surface is about one order, and hence we set $\varsigma_{1}=10$. The distance from the peak position to a concentration of $1 / 10$ of peak concentration is simply evaluated as $70.6 \mathrm{~nm} . \alpha_{1}$ is determined as 2.38 so as to fit the profile in this region. It should be noted that the fixed points in the figure are 


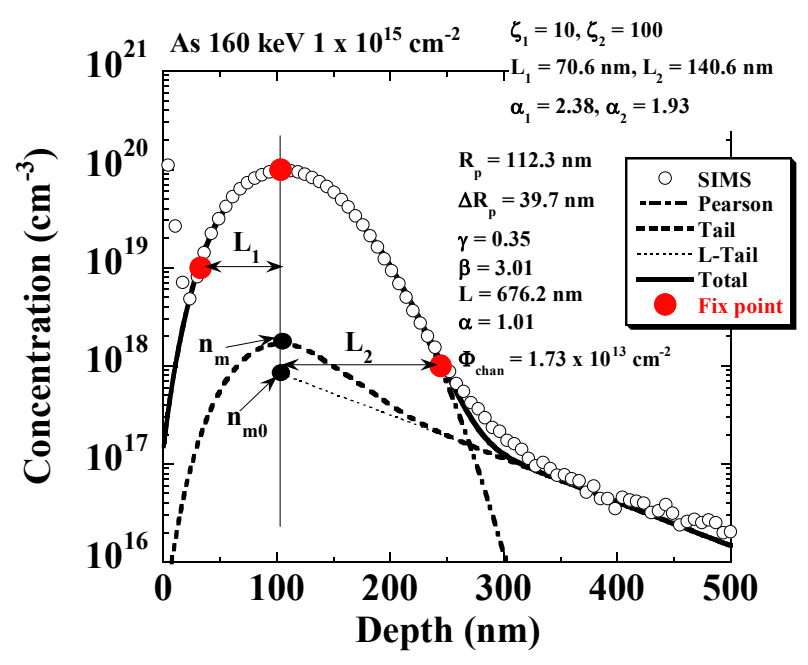

Fig. 12. Comparison of SIMS As data with Pearson function generated using moment parameters from joined tail function acting as mediate function.

invariable during $\alpha_{1}$ tuning. A channeling tail emerges where the concentration is $1 / 100$ of the peak concentration, and hence we set $\varsigma_{2}=100$, and $L_{2}$ is easily determined to be $140.6 \mathrm{~nm}$, and $\alpha_{2}$ to be 1.93 . We can thereby generate the Pearson profile shown in Fig. 12.

We then focus on the profile deeper than $350 \mathrm{~nm}$, and fit the profile with the single tail function (denoted by Ltail in the figure) by tuning $L, \alpha$, and $n_{m 0}$. The corresponding parameters for construction of the tail function can then be calculated automatically using Eqs. $(33,34)$ and the whole profile can be generated as shown in the figure. The evaluated $\Phi_{\text {chan }}$ was $1.73 \times 10^{13} \mathrm{~cm}^{-2}$. The other parameters are also shown in the figure.

\section{SUMMARY}

We showed that the third and fourth moment parameters of the Pearson function are difficult to extract uniquely. We proposed a parameter extraction procedure for the Pearson function by using a joined tail function as mediator. The moment parameters extracted from the mediate function are applied so as to generate the Pearson function. We further proposed a simple procedure to extract the tail function parameters associated with channeling phenomena. All of the parameters used for the extraction are directly related to the shape of the profiles, and hence can be uniquely determined. Using this extraction procedure, we can construct a robust ion implantation database based on the tail function.

\section{REFERENCES}

[1] J. F. Gibbons, S. Mylroie, "Estimation of impurity profiles in ion-implanted amorphous targets using joined half-Gaussian distributions," Appl.Phys.Let., Vol.22, p.568, 1973.

[2] W. K. Hofker, "Implantation of boron in silicon," Philips Res. Rep. Suppl., Vol.8, pp.1-121, 1975.

[3] D. G. Ashworth, R. Oven, and B. Mundin, "Representation of ion implantation profiles by Pearson frequency distribution curves," Appl. Phys. D., Vol.23, pp.870-876, 1990.

[4] A. F. Tasch, H. Shin, C. Park, J. Alvis, and S. Novak, "An improved approach to accurately model shallow B and BF2 implants in silicon," $J$. Electrochem. Soc., Vol.136, pp.810-814, 1989.

[5] C. Park, K. M. Klein, and A. L. Tasch, "Efficient modeling parameter extraction for dual Pearson approach to simulation of implanted impurity profiles in silicon," Solid-State Electronic, Vol.33, pp.646-650, 1990.

[6] K. Suzuki, Ritsuo Sudo, and T. Feudel, "Simple analytical expression for dose dependent ionimplanted Sb profiles using a jointed half Gaussian and one with exponential tail," Solid-State Electronics, Vol.42, pp.463-465, 1998.

[7] K. Suzuki, R. Sudo, Y. Tada, M. Tomotani, T. Feudel, and W. Fichtner, "Comprehensive analytical expression for dose dependent ion-implanted impurity concentration profiles," Solid-State Electronic, Vol.42, pp.1671-1678, 1998.

[8] K. Suzuki and R. Sudo, "Analytical expression for ion-implanted impurity concentration profiles," Solid-State Electronics, Vol.44, pp.2253-2257, 2001.

[9] FabMeister-IM: http://www.mizuho-ir.co.jp/solution/ research/semiconductor/fabmeister/ion/index.html

[10] Sentaurus Process: http://www.sysnopsys.com/Tools/ TCAD/ProcessSimulation?Pages?SentauruProcess. aspx. 


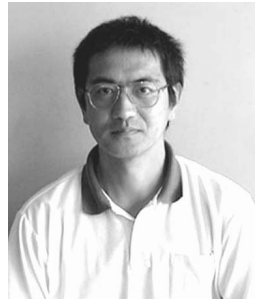

Kunihiro SUZUKI received B. S., M. S., and Ph. D. degrees in electronics engineering from Tokyo Institute of Technology, Tokyo, Japan, in 1981, 1983, and 1996, respectively. He joined Fujitsu Laboratories Ltd., Atsugi, Japan, in 1983 and has been engaged in design and modeling of high-speed bipolar and MOS transistors. $\mathrm{He}$ was a visiting researcher at the Swiss Federal Institute of Technology (ETH) Zurich, Switzerland in 1996 and 1997, where he studied process modeling. From 2006 to 2007, He was with new transistor structure group in MIRAI project, where he studied Ge MIS transistors. His current interests are process and device modeling. He is a senior member of IEEE.

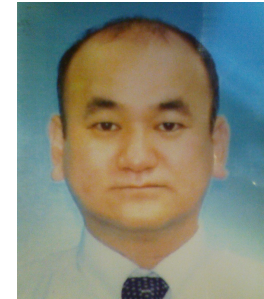

Shuichi KOJIMA received a B. S. degree in applied chemistry from Kanagawa University in 1990. He joined Fujitsu LSI Technology Ltd., Kawasaki, Japan, in 1990 and has been engaged in developing process simulator and manufacture execution system. $\mathrm{He}$ is currently with the same division in Akiruno, and his current interests are developing a process simulator and manufacture scheduling system. 